\title{
Surface Self-Diffusion by Vacancy Motion: Island Ripening on Cu(001)
}

\author{
J. B. Hannon, * C. Klünker, M. Giesen, and H. Ibach \\ Institut für Grenzflächenforschung und Vakuumphysik, Forschungszentrum Jülich, D-52425, Jülich, Germany
}

\author{
N. C. Bartelt and J. C. Hamilton \\ Sandia National Laboratories, Livermore, California 94551
}

(Received 14 April 1997)

\begin{abstract}
We have used scanning tunneling microscopy to study the Ostwald ripening of 2D islands of $\mathrm{Cu}$ grown on $\mathrm{Cu}(001)$. By considering the time dependence of the sizes of individual islands we have characterized the mechanisms for the ripening. Our result is unexpected for a simple metal surface: The flow of atoms from one island to another is limited by attachment-detachment kinetics at the island edges. To explain this result, we propose that the transport of atoms between islands occurs by vacancy, rather than by adatom, diffusion. [S0031-9007(97)04112-4]

PACS numbers: 68.35.Fx, 61.16.Ch, 82.65.Dp
\end{abstract}

It is relatively easy to catalog the many atomic processes possibly involved in surface self-diffusion. It is much harder to quantify them experimentally or to determine which particular processes govern surface evolution on a large length scale. A basic process to consider is an adatom diffusing on a terrace towards a step edge, where the adatom is incorporated. On a simple metal surface there are no obvious additional barriers for this incorporation if the adatom approaches the step from below: The barrier for diffusion on the terrace will be the same or larger than the barriers associated with incorporation into the step edge. It is thus generally believed, and has been observed in 2D island decay on $\mathrm{Ag}(111)$ [1] and $\mathrm{Cu}(111)$ [2], that the flow of atoms to or from step edges is limited by the rate at which adatoms diffuse on the terraces. Since it is often assumed that surface morphology equilibrates by surface steps exchanging adatoms, this belief is central to models of surface self-diffusion which are detailed enough to take surface steps into account [3]. In this paper we will show, however, that mass transfer between islands on $\mathrm{Cu}(001)$ is not diffusion limited over a range of temperatures: Rather it is limited by the attachment and detachment processes at step edges. We propose that this occurs because surface self-diffusion on $\mathrm{Cu}(001)$ occurs by vacancy, rather than by adatom, diffusion.

To probe the mechanisms of diffusion between step edges we have used scanning tunneling microscopy (STM) to measure the time dependence of the sizes of 2D $\mathrm{Cu}$ islands grown on $\mathrm{Cu}(001)$. The deposited islands are out of equilibrium: Large islands grow at the expense of small islands to reduce the total step length and thus the free energy of the system. By studying this ripening process it is possible to extract quantitative information on the kinetics of mass transport at the surface: 2D island ripening provides a geometry which can be readily analyzed because the driving force for adatom diffusion and step motion (step tension and curvature) can be determined from the island shapes and sizes.
In the standard theory [4-6] of island ripening by adatom motion, the time rate of change of the area of an island is determined by two kinetic processes: the diffusion of adatoms on the surrounding terrace towards or away from the island, and the transfer of atoms onto, or off, the island edge. The diffusion rate on the terraces is determined by the adatom diffusion coefficient $D$. The transfer rate between the island edges and the surrounding adatom sea is determined by the attachment rate of adatoms. We express this rate as $\kappa D c$, where $c$ is the adatom concentration next to the step edge and $\kappa$ is a step edge incorporation coefficient. In the near equilibrium process of island ripening, the detachment rate will be close to the attachment rate. Since the rate of atoms moving into any particular empty site on the terrace is proportional to $D c$, $\kappa$ is a measure of the difference in the diffusion barrier for adatoms attaching to a step edge compared to diffusion on the terrace. Depending on whether $\kappa$ is small or near unity, island ripening will be "attachment-detachment limited" or "diffusion limited," respectively.

For the pure attachment-detachment-limited case, concentration gradients in the adatom sea are very small; the adatom concentration surrounding every island assumes a value $c$ which is determined by a balance between the emission and adsorption rates of many islands. In this limit, the rate of change of the area $A$ of an island labeled with index $i$ can be expressed in terms of the difference between $c$ and the concentration $c_{i}^{\text {eq }}$ of adatoms which is needed to establish equilibrium with this island [5-7]: $d A_{i} / d t=C_{i} D \kappa a\left(c-c_{i}^{\mathrm{eq}}\right)$, where $C_{i}$ is the island circumference, and $a$ is the linear dimension of an adatom. If an island has its equilibrium shape, the dependence of $c_{i}^{\mathrm{eq}}$ on island size is given by the GibbsThomson equation for a 2D island on a (001) substrate [8]: $c_{i}^{\mathrm{eq}}=c_{\infty} \exp \left(a^{2} \beta^{[110]} / k T r_{i}^{[110]}\right)$, where $\beta^{[110]}$ is the free energy per unit length of steps along the [110] direction, $r_{i}^{[110]}$ is the radius of the island perpendicular to that orientation, and $c_{\infty}$ is the equilibrium adatom 
concentration on an island-free terrace. Defining $\alpha$ by $C_{i}=\alpha r_{i}^{[110]}$, and assuming $r_{i}^{[110]} \gg \beta^{[110]} a^{2} / k T$, gives

$$
\frac{d A_{i}}{d t}=\frac{\alpha \beta^{[110]} \kappa D a^{3} c_{\infty}}{k T} r_{i}^{[110]}\left(\frac{1}{r_{c}}-\frac{1}{r_{i}^{[110]}}\right) .
$$

The critical radius $r_{c}$ determines which islands grow or shrink. It depends on $c$, and if one can ignore the bounding straight steps can be computed by considering the implications of global mass conservation: $\sum_{i} d A_{i} / d t=0$ implies $r_{c}=\left\langle r^{[110]}\right\rangle$ [9]. So, when $r^{[110]} \ll\left\langle r^{[110]}\right\rangle$, the decay rate will be constant and the island area will decrease linearly in time. For larger islands, which have radii closer to $\left\langle r^{[110]}\right\rangle$, the decay will be slower. Islands with radii larger than $\left\langle r^{[110]}\right\rangle$ will grow.

The effect of diffusion in the adatom sea on Eq. (1) can be determined by solving the diffusion equation for the steady state adatom currents away from each island. For the case of circular islands with radii $r_{i}$ and an isotropic step free energy per unit length $\beta$, the rate of change of each island area has the form $[5,6]$,

$$
\frac{d A_{i}}{d t}=\frac{2 \pi \beta D a^{4} c_{\infty}}{k T} \frac{1}{\ln \left(l_{i}\right)+a / \kappa r_{i}}\left(\frac{1}{r_{c}}-\frac{1}{r_{i}}\right),
$$

again assuming that $r_{i} \gg \beta a^{2} / k T$. The length $l_{i}$ specifies the distance over which the concentration decays to its value far from the step edge, in units of the island radius. In general, $l_{i}$ depends on the spatial configuration and sizes of islands surrounding each island. Although $r_{c}$ is determined by global mass conservation, as it is in the attachment-limited case, it is now not generally the average island radius.

Equation (2) reproduces the form of Eq. (1) only when $\kappa \ll a / r$. In contrast to the linear decay of small islands in the attachment-limited case of Eq. (1), Eq. (2) yields an island area which decreases as $-t^{2 / 3}$ in the pure diffusionlimited case of $\kappa \gg a / r$.

We now discuss the STM experiments which allow us to distinguish the attachment-detachment- and diffusionlimited scenarios. The microscope used in these experiments is based on the Besocke design [10,11]. The $\mathrm{Cu}(001)$ surface was prepared by sputtering for 20 minutes with $500 \mathrm{eV} \mathrm{Ne}^{+}$ions, followed by 20 minute annealing periods at $1100 \mathrm{~K}$. Following each annealing cycle, the sample was cooled slowly to room temperature. Epitaxial $\mathrm{Cu}$ islands were created by evaporating $\mathrm{Cu}$ onto the surface held at a fixed temperature. The experimental data, in the form of island sizes as a function of time, were obtained by repeatedly scanning a fixed area of the surface at intervals of 1-2 minutes. The images were recorded using a tunneling current of $1.0 \mathrm{nA}$ with the tip biased negatively by 0.10 to $0.70 \mathrm{~V}$ with respect to the sample.

To probe the decay of islands in a number of different local environments, we first examined the decay of small islands $\left(10^{3}\right.$ atoms) grown on top of larger ones $\left(10^{5}\right.$ atoms). Some of the configurations studied are illustrated in the upper panels of Fig. 1. This geometry was created by first evaporating $\mathrm{Cu}$ at $420 \mathrm{~K}$, and then at $343 \mathrm{~K}$. The areas of most of the islands decrease with time as plotted in the lower panel of Fig. 1. When the islands become small, the island area decreases approximately linearly in $t$, consistent with attachment-detachment kinetics but inconsistent with the time dependence characteristic of simple diffusion-limited island decay. Furthermore, the island decay rates when the islands are small are all approximately equal, despite the fact that the local geometries vary greatly. This is again most consistent with attachment-detachment-limited step kinetics.

However, when the areas of the islands are large, Fig. 1 shows that significant deviations from a constant decay rate can occur. Large islands tend to decay more slowly than small islands. This could be evidence of the breakdown of attachment-detachment-limited kinetics. To be confident of a conclusion of attachment-detachment-limited kinetics, this deviation from linearity needs to be accounted for. Linear island decay for attachment-detachment-limited kinetics holds only when $r^{[110]} \ll\left\langle r^{[110]}\right\rangle$ in Eq. (1). The decay rates decrease when $r^{[110]}$ becomes comparable to $\left\langle r^{[110]}\right\rangle$. To check whether a noninfinite $\left\langle r^{[110]}\right\rangle$ plays a role in our measurements, we examined the time dependence of the simpler island configuration shown in Fig. 2 of one terrace containing many islands, where the average radius is better defined. The figure shows STM images of the ripening of an assembly of about 60 islands at a substrate temperature of $343 \mathrm{~K}$. The overall number of atoms is roughly conserved so large islands are growing at the expense of small ones, although some material is going to the bounding step edge. When the islands become small,
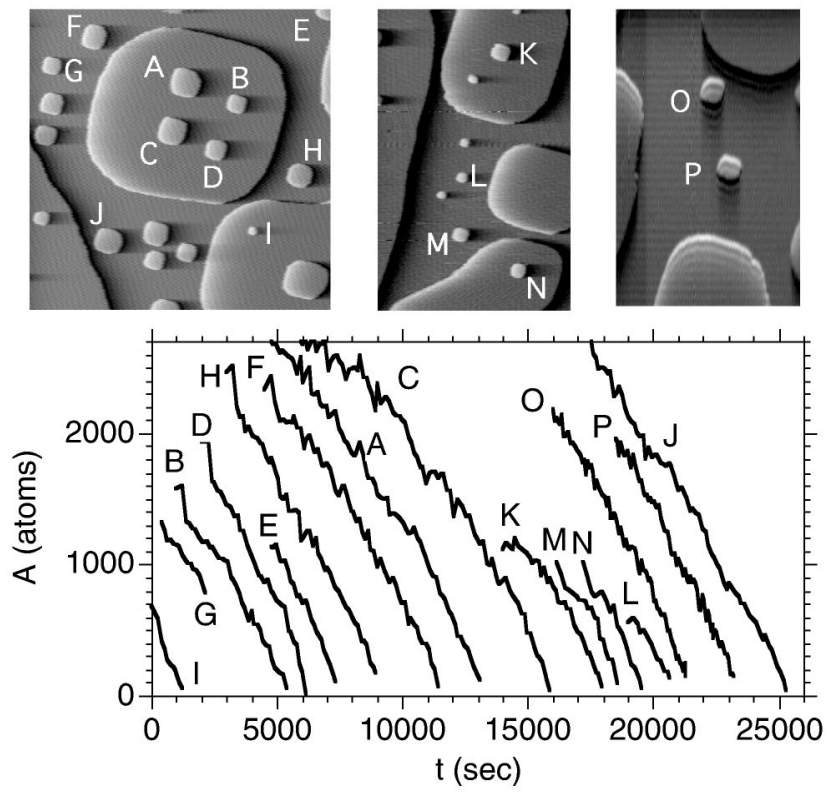

FIG. 1. The upper panels contain STM images of single atomic layer islands grown on $\mathrm{Cu}(001)$ (vertical image dimension is $150 \mathrm{~nm}$ ). The images are differentiated, with white indicating an increase in slope from left to right. The lower panel shows the time dependence of the areas of the labeled islands. These curves have been arbitrarily shifted in time. 

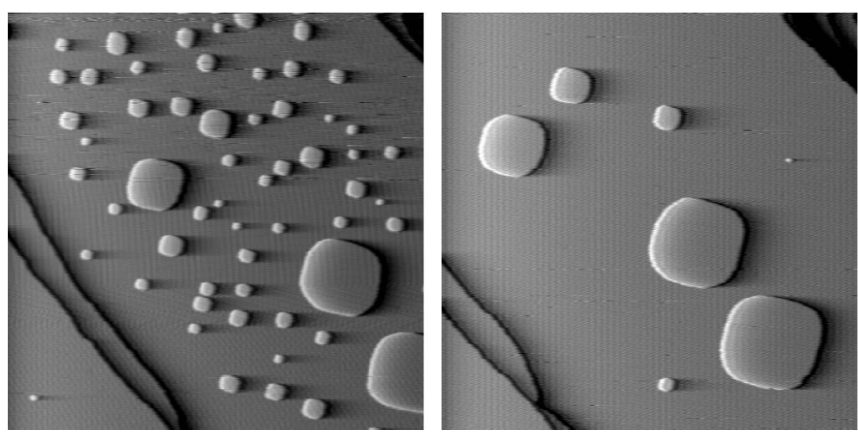

FIG. 2. Two $300 \mathrm{~nm} \times 300 \mathrm{~nm}$ STM images, separated in time by $20000 \mathrm{~s}$, showing island ripening on $\mathrm{Cu}(001)$ at $343 \mathrm{~K}$.

they are observed to randomly move relative to the large islands. Occasionally, as a result of this motion, a small island will coalesce with a larger island. This process, however, occurs only rarely, and only after the areas of the small islands have decreased. The shape of the islands is always the same, even shortly after coalescence, suggesting that edge diffusion is fast enough for the island shapes to have their equilibrium form [12].

To see if this observed ripening behavior is consistent in detail with the attachment-detachment-limited case of Eq. (1), we computed the time dependence of the island areas predicted from Eq. (1), starting from the initial island configuration shown in Fig. 2. In this calculation, the value of $2 \pi D \kappa \beta^{[110]} a^{3} c_{\infty} / k T$ was obtained from fits to a linear island decay rate when the island radii were small. The value of $\left\langle r^{[110]}\right\rangle$ was obtained by averaging over all remaining islands at each time. Figure 3(a) compares the entire configuration predicted from Eq. (1) (white) with experiment for a few selected times. Figure 3(b) compares the calculated time dependence of several islands with the observed behavior. Equation (1) accounts well for the time dependence of almost all of the islands. In particular, Fig. 3(b) shows that it describes, without any adjustable parameters, the range of island areas where nonlinear decay is observed. The island labeled $A$ in Fig. 3(a), which has a predicted area much smaller than observed in experiment, suffered a coalescence event at an early time.

On the other hand, the diffusion-limited prediction works less well. Figure 3(c) compares experiment with the time dependence predicted from Eq. (2), taking $\kappa \approx$ 1. For simplicity, $l$ was assumed to be independent of the island radii. The value of $\alpha D \kappa \beta^{[110]} a^{4} c_{\infty} / \ln (l) k T$ was chosen to give the overall best fit to the observed time dependence of the selected islands. The value of $r_{c}$ at each time was determined by requiring that the sum of all island areas was constant. The diffusion-limited case does not account as well for the overall time dependence of the configuration. In addition, it fails for small islands, where the calculation gives $t^{2 / 3}$ behavior while the experiment shows almost linear decay in time.

As pointed out by Morgenstern et al. [1], the arguments leading to Eq. (2) contain assumptions which break down when the island radii become small, leading to a failure
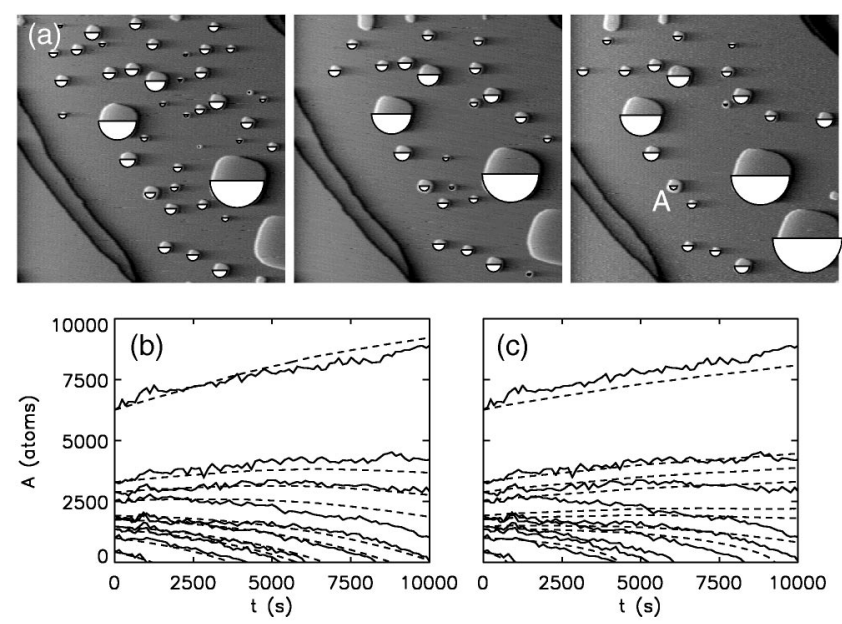

FIG. 3. (a) A comparison of the experimental island configurations at 3000, 6000, and $9000 \mathrm{~s}$ with the predictions of Eq. (1) (white semicircles). In (b) the solid lines show the experimental time dependence of several of the islands areas; the dashed lines show the predictions of Eq. (1). The dashed line in (c) shows the less successful predictions of Eq. (2).

of the $t^{2 / 3}$ law. One might be tempted to invoke such effects to explain the breakdown of Eq. (2). However, these effects tend to give effective power laws for decay which are smaller than $2 / 3$ and thus cannot explain the linear behavior we observe. Another possible objection to our use of Eq. (2) is that in comparing it to experiment we have neglected the effects of the local environment which are encompassed in $l$. However, as shown in Fig. 1, we see no evidence for such effects. (In contrast, in experiments on $\mathrm{Cu}(111)$ [2], where ripening is diffusion limited, effects of the island environment are clearly evident.) We thus conclude that island ripening on $\mathrm{Cu}(001)$ is inconsistent with diffusion-limited mass transport and consistent with attachment-detachment-limited kinetics. This result is not peculiar to $343 \mathrm{~K}$ : In experiments performed in the temperature range from 343 to $413 \mathrm{~K}$ we also find that the decay is attachment-detachment limited.

As was stated above, it is often assumed that simple materials should exhibit diffusion-limited ripening behavior, so the conclusion that attachment-detachment kinetics limits mass transport on $\mathrm{Cu}(001)$ is unexpected. The rationale behind the general belief is that there is no obvious mechanism which will lead to a larger barrier to the attachment of diffusing adatoms at a step edge from below compared to the diffusion barrier on a terrace [6]. Indeed, one expects $\kappa \approx 1$ since an adatom hopping onto a step edge will have a higher coordination as it moves than an adatom diffusing on a terrace. This increased coordination will lower the barrier to adatom attachment and detachment, both for adatoms diffusing by bridge hopping and by place exchange. Thus the diffusion-limited decay of islands seen on $\mathrm{Ag}(111)$ [1] and $\mathrm{Cu}(111)$ [2] is straightforward to understand in terms of adatom motion. To date, systems where attachment-detachment-limited step kinetics have been observed can be explained by the presence 
of a complex surface unit cell $[7,13]$, which requires the coordinated motion of many adatoms for a single incorporation event, or by the presence of adsorbates [14]. No such mitigating factors appear to exist for clean $\mathrm{Cu}(001)$.

We propose that the island ripening on $\mathrm{Cu}(001)$ is attachment-detachment limited because surface vacancies, rather than adatoms, are primarily responsible for mass transport between island edges. For mass transport to be attachment-detachment limited, $\kappa$ in Eq. (1) must be small: The barrier for incorporation of the diffusing species into the step edge must be larger than the barrier for diffusion on the terraces. Vacancy incorporation into a step edge from below involves an annihilation event such as the event at a kink site pictured in Fig. 4. Such annihilation processes have intermediate states (the lower left panel of Fig. 4) which have a lower net coordination than the intermediate states involved in vacancy diffusion on the terraces, leading to a larger barrier for vacancy incorporation [15]. This situation is similar to the states of decreased coordination which adatoms experience when they approach a step edge from above, and leads to the Schwoebel barrier for adatoms. So, in principle, vacancy diffusion could account for our observation of attachment-detachment-limited island ripening. We expect the existence of a large vacancy incorporation barrier to be independent of the details of the atomic potential. To give an indication of the size of the incorporation barrier, we have performed energetics calculations with empirical potentials for $\mathrm{Cu}$. Figure 4 shows the increased diffusion barrier for a vacancy approaching a kink site using the potential obtained from the embedded atom method (EAM). The barrier for vacancy diffusion on the terraces, $E_{v}^{\mathrm{diff}}$, is $0.44 \mathrm{eV}$; the barrier associated with the final annihilation event, $E_{v}^{\text {attach }}$, is $0.62 \mathrm{eV}$ and hence significantly larger.
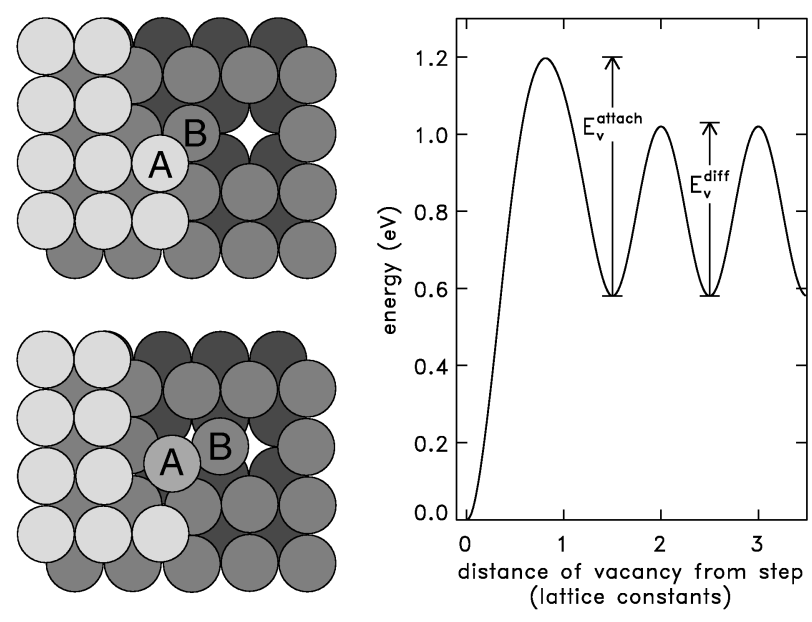

FIG. 4. The left panel shows a vacancy being incorporated into a step edge at a kink site: Atom $B$ moves into the vacancy and atom $A$ drops down to replace $B$. The right panel shows the diffusion barriers experienced by a vacancy as it approaches a kink, based on an EAM calculation for $\mathrm{Cu}(001)$.
In summary, vacancy diffusion provides a plausible explanation for the otherwise unexpected attachmentdetachment ripening we observe on $\mathrm{Cu}(001)$. From simple bond counting arguments, vacancies and adatoms on terraces will have comparable formation energies and diffusion constants on many surfaces. Thus whether vacancy densities and diffusion coefficients are sufficiently large to compete with adatom processes will be sensitive to the detailed balance of relative energetics of vacancies and adatoms. Since the arguments of this paper suggest that vacancies can fundamentally alter the way in which surface morphology equilibrates, this balance needs to be carefully considered in interpreting experiments on surface evolution.

We thank M. Bartelt, G. Rosenfeld, and B. Voigtländer for useful discussions.

Note added. - Further evidence for the importance of vacancy diffusion comes from recent $a b$ initio calculations by G. Boisvert and L. J. Lewis [Phys. Rev. B (to be published)] which suggest that vacancies diffuse more quickly than adatoms on $\mathrm{Cu}(100)$.

*Present address: Sandia National Laboratories, Albuquerque, NM 87185.

[1] K. Morgenstern, G. Rosenfeld, and G. Comsa, Phys. Rev. Lett. 76, 2113 (1996).

[2] G. Schulze Icking-Konert, M. Giesen, and H. Ibach (to be published).

[3] A. Rettori and J. Villain, J. Phys. (France) 49, 257 (1988).

[4] P. Wynblatt and N. A. Gjostein, in Progress in Solid State Chemistry, edited by J.O. McCaldin and G. Somorjai (Pergamon, Oxford, 1975), Vol. 9, p. 21.

[5] M. Zinke-Allmang, L. C. Feldman, and M.H. Grabow, Surf. Sci. Rep. 16, 377 (1992).

[6] J. G. McLean, B. Krishnamachari, D. R. Peale, E. Chason, J. P. Sethna, and B.H. Cooper, Phys. Rev. B 55, 1811 (1997).

[7] N. C. Bartelt, W. Theis, and R. M. Tromp, Phys. Rev. B 54, 11741 (1996).

[8] C. Herring in The Physics of Powder Metallurgy, edited by W.E. Kingston (McGraw-Hill, New York, 1951).

[9] If terrace diffusion is not infinitely fast, then the average should be taken only over a finite region [7].

[10] K. Besocke, Surf. Sci. 181, 145 (1987).

[11] J. Frohn, J. F. Wolf, K. Besocke, and M. Teske, Rev. Sci. Instrum. 60, 1200 (1989).

[12] More evidence that islands have equilibrium shapes comes from fitting the shapes to models as in M. Breeman, G. T. Barkema, and D. O. Boerma [Surf. Sci. 323, 71 (1995)]. These fits give a step kink energy of $1850 \pm$ $400 \mathrm{~K}$, which agrees with the $1486 \pm 40 \mathrm{~K}$ measured by M. Giesen-Seibert et al. [Surf. Sci. 329, 47 (1995)].

[13] L. Kuipers, M. S. Hoogeman, and J. W. M. Frenken, Phys. Rev. Lett. 71, 3517 (1993).

[14] D. R. Peale and B. H. Cooper, J. Vac. Sci. Technol. A 10, 2210 (1992).

[15] T. Michely, T. Land, U. Littmark, and G. Comsa, Surf. Sci. 272, 204 (1992). 